\title{
Carpal Tunnel Syndrome As a Presenting Feature of Polymyositis: A Case Report From India
}

\author{
Arijit NAG, ${ }^{1}$ Debanjali SINHA, ${ }^{1}$ Sumantro MONDAL, ${ }^{1}$ Atanu CHAKRABORTY, ${ }^{1}$ Alakendu GHOSH ${ }^{2}$ \\ ${ }^{1}$ Department of Medicine, The Institute of Post Graduate Medical Education \& Research, Kolkata, India \\ ${ }^{2}$ Department of Medicine and Rheumatology, The Institute of Post Graduate Medical Education \& Research, Kolkata, India
}

\begin{abstract}
Polymyositis is a subset of idiopathic inflammatory muscle disorders in which clinical spectrum includes involvement of predominantly musculoskeletal system with varying degree of gastrointestinal, pulmonary and cardiac manifestations. Neuropathic presentation is extremely unusual. In this article, we report a very rare case of a 33-year-old female case with polymyositis with bilateral carpal tunnel syndrome.

Keywords: Carpal tunnel syndrome; myositis; polymyositis.
\end{abstract}

Inflammatory muscle diseases are a heterogeneous group of systemic autoimmune disorders characterized by chronic muscle weakness, muscle fatigue, and histologically mononuclear cell infiltration into the skeletal muscle. Weakness of the shoulder and hip girdle muscle constitutes one of the predominant presenting features of polymyositis with arthritis (affecting small joints, in particular), skin rashes, constitutional features (fever, fatigue, and malaise, etc.), and cardiac, gastrointestinal, and pulmonary manifestations in a wide range of patient subsets. On the other hand, polymyositis may occur in the absence of muscle pain or constitutional symptoms. It may present in an acute, subacute, or chronic insidious form. ${ }^{1}$ Although rare, carpal tunnel syndrome (CTS) may also develop in the presence of inflammatory myositis. In the literature, CTS which was detected incidentally during nerve conduction studies (NCS) in few cases of inflammatory myositis was reported with an estimated prevalence of $8 \% .^{2}$ In our case, the predominant symptom was proximal muscle weakness of all four limbs, which was associated with a tingling sensation and weakness of distal muscles of the hands. The patient was eventually diagnosed with polymyositis with bilateral CTS.

\section{CASE REPORT}

A 33-year-old nondiabetic, nonhypertensive woman presented with a complaint of proximal muscle weakness of all four limbs for the past two months. She also complained of paresthesia in both palms, more on the radial side, which increased with cold exposure and gripping objects in the palm. She had a history of Raynaud's phenomenon with evidences of digital infarcts (Figure 1), fatigue, malaise, and low-grade fever $\left(37.7-43.3^{\circ} \mathrm{C}\right)$ for two months. The patient had neither a history of trauma to the hands or of upper limb surgery, or hypothyroidism nor statin or corticosteroid or any other drug known to cause myopathy. Her family history was nonspecific.

Received: August 27, 2013 Accepted: December 16, 2013

Correspondence: Sumantro Mondal, M.D. Department of Medicine, Institute of Post Graduate Medical Education and Research, 700020 Kolkata, India.

Tel: 9733852826 e-mail: drmsumantro@gmail.com

(2014 Turkish League Against Rheumatism. All rights reserved. 
Physical examination revealed proximal muscle weakness of the upper and lower limbs (muscle test 3/5), and weakness of the distal muscles of both hands, in the abductor pollicis brevis, opponens pollicis, flexor pollicis and the first and second lumbricals particularly. The Phalen's test (Figure 2), reverse Phalen's test and Tinel's sign were positive. However, the patient was unable to perform the test accurately due to the pain along the bilateral median nerves. All other systemic examination findings were noncontributory, and there were no clinical manifestations of acromegaly.

Her creatine phosphokinase level was $3100 \mathrm{U} / \mathrm{L}$ (normal value less than $195 \mathrm{U} / \mathrm{L}$ ), alanine aminotransferase level was $160 \mathrm{U} / \mathrm{L}$ (normal value less than $40 \mathrm{U} / \mathrm{L}$ ), and aspartate aminotransferase level was $173 \mathrm{U} / \mathrm{L}$ (normal value less than $37 \mathrm{U} / \mathrm{L})$. Rheumatoid factor and U1-RNP were negative, and thyroid function test results were normal. The patient tested positive for anti nuclear antibody with a titer of 1:1000 and speckled pattern (HEp-2). Anti-double-stranded DNA, anticentromere, antitopoisomerase I, antiRo/SSA and La/SSB were negative. The results of the urinalysis and microscopic examination urine were nonspecific and spot albumin-creatinine ratio was 26.40 (normal value less than 30 ).

X-rays of bilateral wrist joints and abdominal ultrasonography were normal. Echocardiography and pulmonary function tests revealed no abnormal pathology. Electromyography showed low amplitude, short duration, polyphasic motor unit potentials with full recruitment, suggesting a myopathic pattern. The motor NCS showed low compound muscle action potential amplitudes and

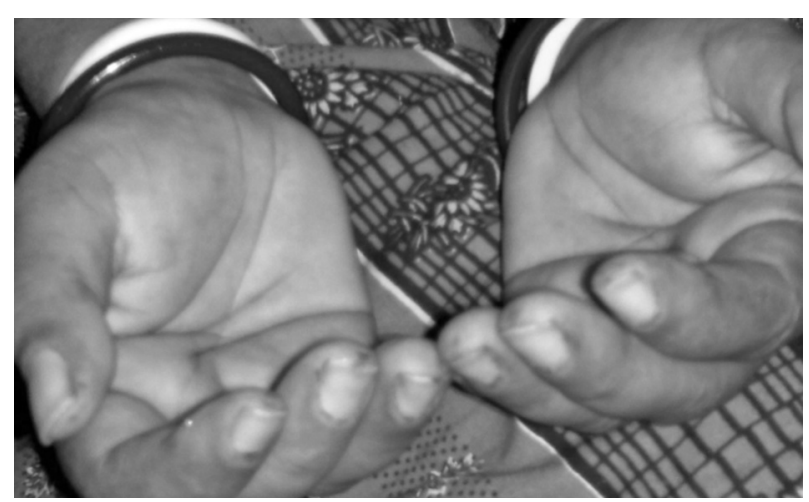

Figure 1. Digital infarct involving both hands of the patient. prolonged distal latencies in both median nerves, and a low compound muscle action potential of the left median nerve of the wrist. Sensory NCSs showed an increased-onset latency in the right median nerve and an unexcitable left median nerve, which are thereby, consistent with bilateral CTS (Table 1). Muscle biopsy revealed muscle fiber necrosis with few muscle fibers showing mononuclear cell infiltration and endomysial infiltration without perifascicular atrophy or any suggestion of vasculitis, which was consistent with polymyositis.

The patient was diagnosed with polymyositis with bilateral CTS, and was started oral corticosteroids (prednisolone $1 \mathrm{mg} / \mathrm{kg}$ ), subcutaneous methotrexate (15 mg weekly) and folic acid (5 mg weekly).

She has been under follow-up for two months with reduced proximal and distal muscle weakness and improved wellbeing.

\section{DISCUSSION}

Polymyositis is an idiopathic inflammatory myositis which manifests itself as symmetrical proximal muscleweakness, elevated muscleenzyme levels, and typical electromyographic and muscle biopsy findings. The disease presents in various modes, the most common being muscle weakness associated with constitutional features (fever, malaise, and fatigue). Pulmonary, cardiovascular, or gastroenterological manifestations or inflammatory arthritis are common in patients

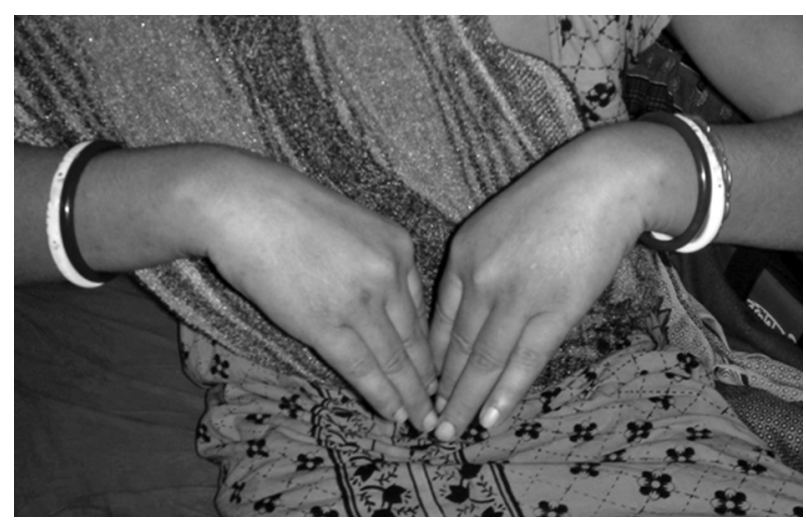

Figure 2. Patient attempting to perform the Phalen's test. However, she was unable to flex her wrist beyond this point due to severe pain along bilateral median nerves. 


\begin{tabular}{|c|c|c|c|}
\hline \multirow[b]{2}{*}{ Nerve } & \multicolumn{3}{|c|}{ Motor nerve conduction study } \\
\hline & Latency (msec) & Amplitude (mV) & $\mathrm{NCV}(\mathrm{m} / \mathrm{s})$ \\
\hline Right median nerve: wrist & 4.9 (normal <4) & 4.9 & 63.06 \\
\hline Elbow & 8.23 & 3.9 & \\
\hline Right ulnar: wrist & 1.77 & 2.4 & 69.07 \\
\hline Elbow & 5.10 & 2.1 & \\
\hline Left median nerve: wrist & 5.94 (normal <4) & 3.3 (normal >4) & 63.06 \\
\hline Elbow & 9.27 & 2.6 & \\
\hline Left ulnar: wrist & 2.29 & 2.8 & 59.59 \\
\hline \multirow[t]{2}{*}{ Elbow } & 6.15 & 2.3 & \\
\hline & \multicolumn{2}{|c|}{ Sensory nerve conduction study } & \\
\hline Nerve & Latency (msec) & Amplitude $(\mu \mathrm{V})$ & \\
\hline Right median nerve: palm-wrist & 4.0 (normal <3) & 12.1 & \\
\hline Right ulnar: $5^{\text {th }}$ digit-wrist & 1.71 & 34.8 & \\
\hline Left median nerve: palm-wrist & Not recordable & Not recordable & \\
\hline Left ulnar: $5^{\text {th }}$ digit-wrist & 1.63 & 38.6 & \\
\hline
\end{tabular}

with polymyositis or dermatomyositis. Peripheral neuropathy may rarely complicate polymyositis. ${ }^{3}$

Carpal tunnel syndrome is an entrapment median neuropathy causing pain, paresthesia, numbness, and other symptoms in the distribution of the median nerve due to its compression at the wrist in the carpal tunnel. ${ }^{4}$ The most common causes of CTS include obesity, use of oral contraceptives, hypothyroidism, arthritis, diabetes, trauma, acromegaly, diabetes, alcoholism, hypothyroidism, kidney failure, menopause, pregnancy, rheumatoid arthritis, systemic lupus erythematosus, and scleroderma. Intrinsic factors exerting pressure within the tunnel, and extrinsic factors (pressure exerted from outside the tunnel), which include benign tumors such as lipomas, ganglion or vascular malformations, may also contribute to the development of CTS.4-6 A study from South India demonstrated that CTS constituted $7 \%$ of all the peripheral nerve disorders, and $83.6 \%$ of entrapment neuropathies. ${ }^{7}$

However, CTS as a presenting feature of polymyositis is extremely rare. The literature review did not reveal symptomatic CTS in cases with polymyositis. Our patient fulfilled the diagnostic criteria for polymyositis. The absence of other possible etiologies and improved hand symptoms after corticosteroid therapy strongly established polymyositis as a causal factor for the development of CTS. To the best of our knowledge, our case presented herein is the first case in literature since CTS was a presenting clinical feature of polymyositis, and it responded to treatment with immunosuppressive medication. This is also a very rare presentation of polymyositis, and the first reported case of such clinical phenotype from India. From a clinical viewpoint, we conclude that CTS may be an important diagnostic checklist item in patients with polymyositis presenting with sensory symptoms in the hand accompanied with distal muscle weakness.

\section{Acknowledgement}

We would like to thank the Director and Medical Superintendant of I.PG.M.E\&R Kolkata, for their support to send the article.

\section{Declaration of conflicting interests}

The authors declared no conflicts of interest with respect to the authorship and/or publication of this article.

\section{Funding}

The authors received no financial support for the research and/or authorship of this article.

\section{REFERENCES}

1. Pearson CM. Polymyositis. Annu Rev Med 1966;17:63-82 
2. Wang Y, Cui LY, Chen L, Liu MS, Qi X, Li BH, et al. Nerve conduction studies in patients with dermatomyositis or polymyositis. Chin Med J (Engl) 2010;123:523-6.

3. Cohen MG, Schwartz MS, Li EK, Kay R. Asymmetrical weakness in polymyositis associated with neuropathic involvement. Clin Rheumatol 1991;10:437-9.

4. Sternbach G. The carpal tunnel syndrome. J Emerg
Med 1999;17:519-23.

5. Katz JN, Simmons BP. Clinical practice. Carpal tunnel syndrome. N Engl J Med 2002;346:1807-12.

6. Tiong WH, Ismael TS, Regan PJ. Fibroma of tendon sheath: a rare cause of carpal tunnel syndrome. J Hand Surg Br 2006;31:579-80.

7. Murthy JMK, Meena AK. Carpal tunnel syndrome-Is it really uncommon in India. Neurol India 1995;43:26-8. 